\title{
MORTALIDADE PERINATAL E DIFERENÇAS REGIONAIS NO ESTADO DO PARANÁ*
}

\author{
Tereza Maria Mageroska Vieira ${ }^{1}$, Rosana Rosseto de Oliveira ${ }^{2}$, Verônica de Azevedo Mazza ${ }^{3}$, Thais Aidar de Freitas Mathias ${ }^{4}$
}

${ }^{1}$ Enfermeira. Mestre em Enfermagem. Docente da Universidade Estadual do Paraná. Paranavaí. PR, Brasil.

Enfermeira. Doutora em Enfermagem. Universidade Estadual de Maringá. Maringá, PR, Brasil.

${ }^{3}$ Enfermeira. Doutora em Saúde Coletiva. Docente de Enfermagem e Programa de Pós-Graduação em Enfermagem da Universidade Federal do Paraná. Curitiba, PR, Brasil.

${ }^{4}$ Enfermeira. Doutora em Saúde Pública. Docente do Programa de Pós-Graduação em Enfermagem da Universidade Estadual de Maringá. Maringá, PR, Brasil.

RESUMO: Objetivou-se analisar a mortalidade perinatal no Estado do Paraná. Foram analisados todos os óbitos perinatais de residentes no Paraná, de 1999-2010, constantes no Sistema de Informação sobre Mortalidade e Sistema de Informações sobre Nascidos Vivos. Foram calculadas as diferenças relativas dos coeficientes de mortalidade perinatal, fetal e neonatal precoce, entre 1999-2001 e 2008-2010 segundo Regionais de Saúde. A análise mostrou redução de $28,2 \%$ no coeficiente de mortalidade perinatal, $32,2 \%$ no neonatal precoce e $25,2 \%$ no fetal. Das Regionais de Saúde, destacaram-se a $21^{\mathrm{a}}$ com aumento de $8,8 \%$ no coeficiente de mortalidade neonatal precoce e $1 \mathrm{a}, 8^{\mathrm{a}}$ e $16^{\mathrm{a}}$ Regionais de Saúde com aumento de 5,9\%, 12,3\% e 12,2\% no coeficiente de mortalidade fetal, respectivamente. Apesar do declínio do coeficiente de mortalidade perinatal para o estado, ainda persistem valores elevados em algumas Regionais de Saúde, o que indica necessidade de aprimorar ações no pré-natal e na atenção ao recém-nascido de risco.

DESCRITORES: Mortalidade perinatal; Mortalidade fetal; Mortalidade neonatal precoce; Sistemas de informação; Enfermagem.

\section{PERINATAL MORTALITY AND REGIONAL DIFFERENCES IN THE STATE OF PARANÁ, BRAZIL}

ABSTRACT: The aim of this study was to assess perinatal mortality in the state of Paraná by analyzing the total number of perinatal deaths among Paraná residents occurring between 1999 and 2010, as registered in the Mortality Information System and Stillborn Information System. This study calculated the differences between perinatal fetal and early neonatal mortality coefficients from 1999 to 2001 and 2008 to 2010, according to data from regional health coordination centers. The results showed a $28.2 \%$ reduction in the perinatal mortality coefficient, $32.2 \%$ in the early neonatal coefficient and $25.2 \%$ in fetal mortality coefficient. Some highlights include a $8.8 \%$ increase in the early neonatal mortality rate reported by the 21 st regional health coordination center, and the $5.9 \%, 12.3 \%$ and $12.2 \%$ increase in the fetal mortality coefficient in the 1st, 8th, and 16th regional centers. Even though the mean state perinatal mortality coefficient fell, high values persist in some regional health centers, pointing to the need for improving actions in prenatal and high-risk neonate care.

DESCRIPTORS: Perinatal mortality; Fetal mortality; Early neonatal mortality; Information systems; Nursing.

\section{MORTALIDAD PERINATAL Y DIFERENCIAS REGIONALES EN EL ESTADO DE PARANÁ}

RESUMEN: El objetivo del estudio fue analizar la mortalidad perinatal en el estado de Paraná. Fueron analizados todos los óbitos perinatales de residentes en Paraná, de 1999-2010, constantes en el Sistema de Información sobre Mortalidad y Sistema de Informaciones sobre Nacidos Vivos. Fueron calculadas las diferencias relativas de los coeficientes de mortalidad perinatal, fetal y neonatal precoz, entre 19992001 y 2008-2010 según Regionales de Salud. El análisis mostró reducción de $28,2 \%$ en el coeficiente de mortalidad perinatal, $32,2 \%$ en el neonatal precoz y $25,2 \%$ en el fetal. De las Regionales de Salud, se destacaron la $21^{\text {a }}$ con elevación de $8,8 \%$ en el coeficiente de mortalidad neonatal precoz y $1 \mathrm{a}$, $8^{\mathrm{a}}$ y $16^{\mathrm{a}}$ Regionales de Salud con elevación de 5,9\%,12,3\% y $12,2 \%$ en el coeficiente de mortalidad fetal, respectivamente. A pesar del declinio del coeficiente de mortalidad perinatal para el estado, aún persisten valores elevados en algunas Regionales de Salud, lo que indica necesidad de perfeccionar acciones en el prenatal y en la atención al recién nacido de riesgo.

DESCRIPTORES: Mortalidad perinatal; Mortalidad fetal; Mortalidad neonatal precoz; Sistemas de información; Enfermería.

*Artigo extraído da dissertação intitulada: "Perfil da mortalidade perinatal no Estado do Paraná no período de 1999 a 2010 ". Universidade Estadual de Maringá, 2013.

Autor Correspondente:

Tereza Maria Mageroska Vieira

Universidade Estadual do Paraná

R. Percy Guimarães Cleves, 1466 - 87704-140 - Paranavaí, PR, Brasil

E-mail: mageroska@yahoo.com.br
Recebido: $14 / 08 / 2015$ Finalizado: 13/10/2015 


\section{INTRODUÇÃO}

A mortalidade perinatal é considerada um indicador de saúde materno-infantil que reflete tanto as condições de saúde, os fatores socioeconômicos, quanto a qualidade da assistência ofertada durante a gestação, parto e ao recém-nascido. A mortalidade perinatal compreende os óbitos ocorridos a partir da $22^{\mathrm{a}}$ semana de gestação até os seis primeiros dias de vida do recém-nascido(1), ou seja, os óbitos fetais somados aos óbitos neonatais precoces.

De acordo com a Organização Mundial de Saúde, a morte fetal ocorre quando o bebê em formação vai a óbito antes da sua expulsão ou da extração completa do corpo da mãe, e esse diagnóstico acontece quando, depois de separado do organismo materno, o feto além de não respirar não apresenta qualquer outro sinal de vida $^{(2)}$.

Estima-se que ocorram no mundo cerca de três milhões de natimortos de terceiro trimestre de gestação a cada ano. Desses óbitos, 98\% ocorrem em países de baixa e média renda, e mais de um milhão de natimortos ocorrem no período intraparto $^{(3)}$.

A taxa de natimortos vem diminuindo com redução de $14,5 \%$ em todo mundo, passando de 22,1 natimortos por mil nascimentos em 1995 a 18,9 natimortos por mil nascimentos em $2009^{(4)}$, sendo que o Brasil manteve-se na média desses índices com 12 natimortos por 1.000 nascimentos ${ }^{(1)}$.

No entanto, a mortalidade fetal apresenta ainda diferenças regionais, com as regiões Norte e Nordeste com as maiores taxas de 13,4 natimortos por mil nascidos em 2006 e a região Sul com a menor taxa de 9,2 natimortos no mesmo

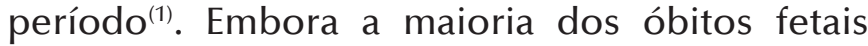
seja evitável, pouca importância tem sido dada ao estudo da sua ocorrência, reflexo da baixa visibilidade, interesse e compreensão de que o óbito fetal pode ser prevenido em grande parte por ações dos serviços de saúde ${ }^{(1)}$.

Em relação à mortalidade neonatal precoce, que ocorre nos primeiros seis dias de vida, a mesma expressa a complexa conjunção de fatores biológicos, socioeconômicos e assistenciais e esses últimos são relacionados à atenção à gestante e ao recém-nascido ${ }^{(5)}$.

No Brasil, existem evidências de maior concentração dos óbitos infantis nos seis primeiros dias de vida. Dos óbitos infantis, cerca de $50 \%$ ocorreram no período neonatal precoce em 2000 e 53\% em 2010. Em 2005, a taxa de óbito neonatal precoce foi de 10,9 por mil nascidos vivos e apesar do esforço para que esta taxa diminua, ainda ocorrem em média 36.000 óbitos neonatais precoces todos os anos no país ${ }^{(6)}$. Da mesma forma, o Estado do Paraná também apresenta para o componente neonatal precoce coeficientes elevados, representados por 6,8 óbitos por mil nascidos vivos em $2010^{(6)}$.

Para o monitoramento da mortalidade perinatal e seus componentes, os Sistemas de Informação em Saúde, desenvolvidos e implantados pelo Ministério da Saúde, constituem ferramentas importantes para a gestão descentralizada do sistema de saúde, e têm apresentado progressiva consolidação e qualificação, fazendo com que tenham seu uso expandido e consolidado nas pesquisas científicas, bem como por profissionais da saúde ${ }^{(7)}$.

Neste cenário, frente à relevância dos óbitos fetais e dos óbitos neonatais precoces na mortalidade infantil, e à importância de identificar situações de desigualdade que demandem ações específicas, além de subsidiar processos de planejamento, gestão e avaliação de políticas e ações de saúde para a atenção pré-natal, ao parto e ao recém-nascido, este estudo teve por objetivo analisar a mortalidade perinatal e seus componentes, no Estado do Paraná, de 1999 a 2010, segundo as Regionais e Macrorregionais de Saúde. Parte-se do princípio de que os coeficientes estejam diminuindo, resultado da expansão da Estratégia Saúde da Família no Estado do Paraná, além das melhorias na situação econômica e social da população como um todo nesta década(8).

\section{MÉTODOS}

Trata-se de um estudo descritivo da mortalidade perinatal e seus componentes (fetal e neonatal precoce) de residentes no Estado do Paraná, ocorridos no período de 1999 a 2010. O Paraná, com população calculada em 10.444.526 habitantes em 2010, tem 399 municípios que estão agrupados em 22 Regionais de Saúde (RS) e quatro Macrorregionais deSaúde.AsRSeMacrorregionais são recortes territoriais identificados para organizar a rede de ações e serviços de saúde a fim de assegurar o cumprimento dos princípios constitucionais de universalidade do acesso, equidade e integralidade do cuidado ${ }^{(9)}$.

Os óbitos foram analisados a partir dos dados do Sistema de Informação sobre Mortalidade 
(SIM) e do Sistema de Informação sobre Nascidos Vivos (Sinasc), com dados disponíveis no sítio do Departamento de Informática do Sistema Único de Saúde (Datasus). Para o cálculo dos coeficientes, foi utilizado o método de cálculo direto de acordo com a metodologia da Rede Interagencial de Informação para a Saúde (RIPSA), que combina dados diretos do SIM/Sinasc dos Estados com boa qualidade de informação ${ }^{(10)}$.

Calcularam-se os coeficientes de mortalidade perinatal considerando o número de óbitos fetais acrescidos dos óbitos neonatais precoces, por mil nascidos vivos (NV) e nascidos mortos (NM). O coeficiente de mortalidade fetal foi calculado considerando o número de óbitos fetais (a partir da $22^{a}$ semana de gestação) por mil nascimentos de mães residentes (NV e NM). O coeficiente de mortalidade neonatal precoce foi calculado considerando o número de óbitos de residentes de zero a seis dias por mil nascidos vivos de mães residentes ${ }^{(10)}$.

Os coeficientes de mortalidade perinatal foram analisados por triênios, levando-se em consideração a disponibilidade dos dados nos Sistemas de Informação (SIM/Sinasc) e os anos censitários. A análise por triênios tem a finalidade de reduzir possíveis flutuações no número de óbitos e nascimentos durante o período. Para a análise da distribuição da mortalidade perinatal, foram calculadas as diferenças relativas dos coeficientes de mortalidade (CM) nos triênios extremos, 1999-2001 e 2008-2010.

A distribuição dos coeficientes de mortalidade perinatal e seus componentes foram analisados por Macrorregionais e Regionais de Saúde nos dois triênios extremos utilizando o mapa do Estado do Paraná, com distribuição dos coeficientes em quartis, de acordo com o valor máximo e mínimo, utilizando-se escalas de cor cinza fixando a cor branca para os coeficientes mais baixos e a cor preta para os coeficientes mais elevados. O estudo respeitou os preceitos éticos de pesquisa envolvendo seres humanos, de acordo com a Resolução 466/12, sendo aprovado em 16 de março de 2012 com o parecer n ${ }^{\circ}$ 5991/2012 pelo Comitê de Ética em Pesquisa envolvendo Seres Humanos da Universidade Estadual de Maringá.

\section{RESULTADOS}

Os resultados mostram que, em geral, ocorreu melhora na saúde da criança residente no estado do Paraná, representada pela queda nos CM perinatal (de 22,8 em 1999 para 15,3 óbitos por mil nascidos vivos e mortos em 2010), e para os dois componentes, neonatal precoce e fetal. Entretanto, embora tenha sido observada queda no período, a Figura 1 mostra oscilação nos coeficientes a partir de 2007, sugerindo tendência de estabilização em valores próximos a 8,6 para o CM fetal e próximo a 6,9 para o CM neonatal precoce.

$\mathrm{Na}$ análise dos coeficientes por regiões do estado, observou-se que os coeficientes de mortalidade perinatal e seus componentes diminuíram para o estado do Paraná, para as Macrorregionais e para a grande maioria das Regionais de Saúde. Para três Regionais de Saúde, houve ligeiro aumento nos coeficientes de natimortalidade, com acréscimo relativo de $+5,9$, $+12,3$ e +12,2, para Paranaguá, Francisco Beltrão e Apucarana, respectivamente (Tabela 1).

Constatou-se que a Macrorregional Noroeste teve redução relativa nos coeficientes de

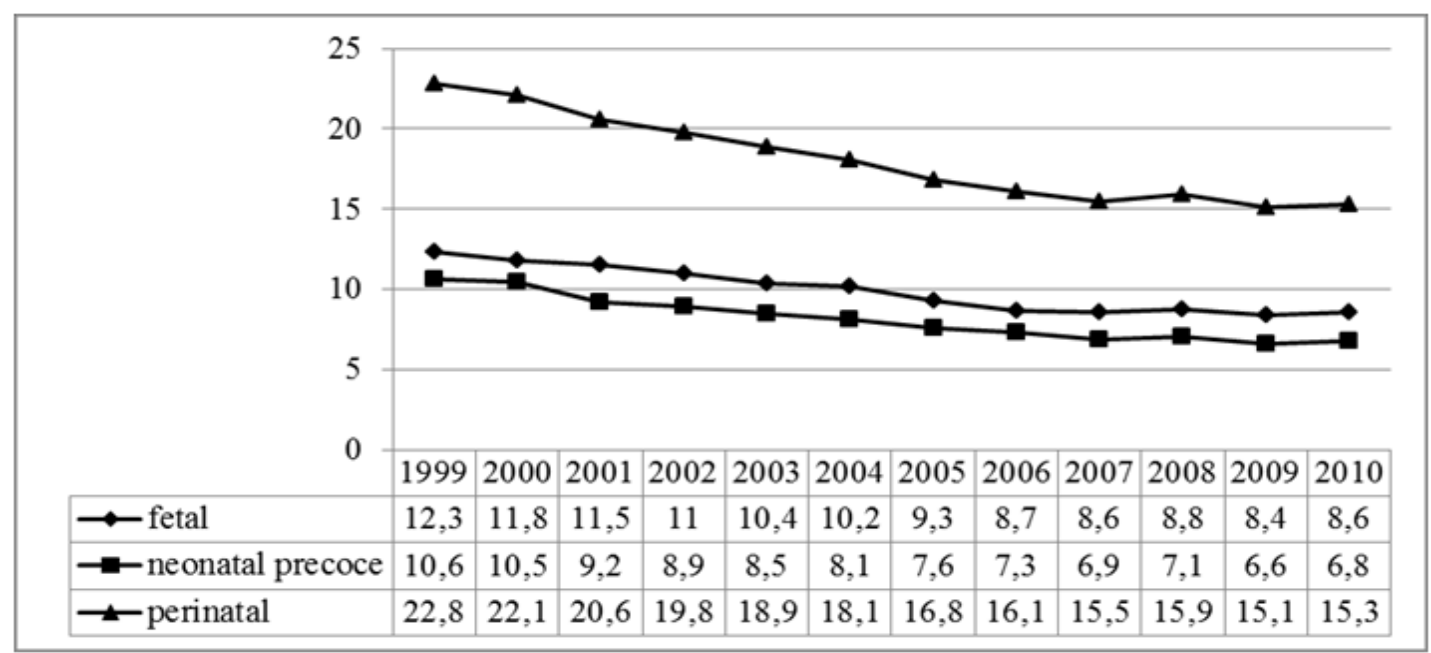

Figura 1 - Coeficiente de mortalidade perinatal, segundo seus componentes. Paraná, Brasil, 1999 a 2010 
Tabela 1 - Coeficientes de mortalidade perinatal, neonatal precoce e fetal, segundo Regional de Saúde e Macrorregional, por triênios. Paraná, Brasil, 1999 a 2001 e 2008 a 2010

\begin{tabular}{|c|c|c|c|c|c|c|c|c|c|}
\hline \multirow[t]{3}{*}{$\begin{array}{l}\text { Macrorregional/ } \\
\text { Regional de Saúde }\end{array}$} & \multicolumn{6}{|c|}{ Triênio } & \multicolumn{3}{|c|}{$\begin{array}{l}\text { Diferença } \\
\text { relativa }(\mathrm{a}-\mathrm{b})\end{array}$} \\
\hline & \multicolumn{3}{|c|}{ 1999-2001 (a) } & \multicolumn{3}{|c|}{ 2008-2010 (b) } & \multirow[b]{2}{*}{ peri } & \multirow[b]{2}{*}{ neo $p$} & \multirow[b]{2}{*}{ fetal } \\
\hline & peri & neo $p$ & fetal & peri & neo $p$ & fetal & & & \\
\hline Leste & 23,5 & 10,6 & 13 & 16,4 & 7,1 & 9,4 & $-30,2$ & -33 & $-27,7$ \\
\hline 1ª Paranaguá & 27,3 & 15,4 & 12,1 & 20,1 & 7,4 & 12,8 & $-26,4$ & -52 & 5,9 \\
\hline $2^{\mathrm{a}}$ Metropolitana & 20,8 & 9,1 & 11,8 & 14,4 & 6,1 & 8,4 & $-30,7$ & -33 & $-29,1$ \\
\hline $3^{\text {a Ponta Grossa }}$ & 27,3 & 13,7 & 13,8 & 19 & 8,3 & 10,8 & $-30,3$ & $-38,8$ & $-22,2$ \\
\hline $4^{\text {a }}$ Irati & 29,4 & 12,6 & 17 & 17,7 & 7,5 & 10,4 & $-39,7$ & $-40,9$ & $-39,2$ \\
\hline $5^{\text {a Guarapuava }}$ & 30,8 & 12,8 & 18,2 & 23,3 & 10,5 & 12,9 & $-24,5$ & -18 & $-29,4$ \\
\hline $6^{\mathrm{a}}$ União Vitória & 19,3 & 9,3 & 10,1 & 12,1 & 3,5 & 8,5 & $-37,1$ & $-62,8$ & $-14,8$ \\
\hline \multirow[t]{2}{*}{ 21a Telêmaco Borba } & 27,3 & 11,3 & 16,2 & 22,4 & 12,3 & 10,1 & -18 & 8,8 & $-36,7$ \\
\hline & peri & neo $p$ & fetal & peri & neo $p$ & fetal & peri & neo $p$ & fetal \\
\hline Oeste & 19,5 & 9,1 & 10,4 & 15 & 7 & 8 & -23 & -23 & $-32,7$ \\
\hline $7^{\text {a }}$ Pato Branco & 20,5 & 9,9 & 10,7 & 17,8 & 8 & 9,7 & $-13,5$ & $-19,5$ & -8 \\
\hline $8^{a}$ Francisco Beltrão & 16,7 & 9,5 & 7,4 & 16,5 & 8,2 & 8,3 & $-1,7$ & $-12,6$ & 12,3 \\
\hline 9a Foz do Iguaçu & 21,5 & 9,4 & 12,2 & 12,9 & 6,3 & 6,6 & -40 & $-33,1$ & $-45,6$ \\
\hline $10^{\mathrm{a}}$ Cascavel & 18,5 & 8,2 & 10,5 & 14,5 & 6,8 & 7,7 & $-21,8$ & $-16,4$ & $-26,2$ \\
\hline \multirow[t]{2}{*}{$20^{\mathrm{a}}$ Toledo } & 20,4 & 9,9 & 10,6 & 15,4 & 6,5 & 8,9 & $-24,6$ & $-33,8$ & $-16,2$ \\
\hline & peri & neo $p$ & fetal & peri & neo $p$ & fetal & peri & neo $p$ & fetal \\
\hline Noroeste & 22,1 & 10,4 & 11,8 & 14,9 & 6,1 & 8,9 & $-32,6$ & $-41,3$ & $-24,6$ \\
\hline $11^{\text {a }}$ Campo Mourão & 24,8 & 11,9 & 13 & 15,7 & 7,2 & 8,6 & $-36,6$ & $-39,2$ & $-34,4$ \\
\hline $12^{\mathrm{a}}$ Umuarama & 22,2 & 10,8 & 11,5 & 15 & 6,4 & 8,7 & $-32,3$ & -41 & $-24,5$ \\
\hline $13^{\mathrm{a}}$ Cianorte & 21,2 & 9,8 & 11,5 & 16,2 & 7,5 & 8,8 & $-23,6$ & $-23,8$ & $-23,7$ \\
\hline $14^{a}$ Paranavaí & 25,8 & 12,2 & 13,7 & 17,9 & 7 & 11 & $-30,5$ & -43 & $-19,6$ \\
\hline \multirow[t]{2}{*}{$15^{\mathrm{a}}$ Maringá } & 19,3 & 8,8 & 10,6 & 13,2 & 4,9 & 8,3 & $-31,8$ & $-44,3$ & $-21,6$ \\
\hline & peri & neo $p$ & fetal & peri & neo $p$ & fetal & peri & neo $p$ & fetal \\
\hline Norte & 19,4 & 8,5 & 11 & 15,7 & 6,7 & 9,1 & $-19,2$ & $-21,2$ & $-17,3$ \\
\hline $16^{a}$ Apucarana & 18,1 & 9,8 & 8,4 & 17,1 & 7,8 & 9,4 & $-5,5$ & $-20,5$ & 12,2 \\
\hline $17^{\mathrm{a}}$ Londrina & 16,7 & 7,4 & 9,3 & 12,4 & 5,6 & 6,9 & $-25,6$ & $-25,1$ & $-26,2$ \\
\hline 18ª Cornélio Procópio & 28,6 & 11,8 & 17,1 & 20,2 & 9,4 & 11 & $-29,6$ & $-20,6$ & $-35,9$ \\
\hline $19^{\mathrm{a}}$ Jacarezinho & 26,8 & 11,9 & 15,1 & 21,9 & 7,2 & 14,9 & $-18,5$ & $-39,5$ & $-2,2$ \\
\hline $22^{\mathrm{a}}$ Ivaiporã & 16,6 & 7,9 & 8,8 & 13,8 & 5,9 & 7,9 & $-17,3$ & $-24,8$ & $-10,8$ \\
\hline Paraná & 22,1 & 10,1 & 12,1 & 15,9 & 6,9 & 9,1 & $-28,2$ & $-32,2$ & $-25,2$ \\
\hline
\end{tabular}

Fonte: Datasus/SIM/Sinasc

mortalidade perinatal de $-32,6 \%$ e a Macrorregional Leste de $-30,2 \%$, mais elevados do que a média do Estado que foi de $-28,2 \%$ (Tabela 1 ).

Comparando-se os coeficientes em cada triênio com a média do estado, destacam-se algumas regiões. No primeiro triênio, duas Macrorregionais, a Norte (19,4 óbitos perinatais /1000) e a Oeste com (19,5 óbitos perinatais 11000) tiveram coeficientes menores do que o do estado do Paraná que foi de 22,1 óbitos perinatais para cada 1000 nascidos vivos e mortos. Já no segundo triênio, três Macrorregionais (exceto a
Macrorregional Leste) tiveram os coeficientes de mortalidade perinatal inferiores ao do estado do Paraná que foi de 15,9 óbitos perinatais por 1000 nascidos vivos e mortos (Tabela 1 ).

No primeiro triênio, as Regionais de Saúde que apresentaram menores coeficientes de mortalidade perinatal foram as RS de Ivaiporã $(16,6)$, Londrina $(16,7)$ e Francisco Beltrão $(16,7)$. No segundo triênio, as RS União da Vitória com coeficiente de 12,1 e ainda Londrina com 12,4 foram as que tiveram menor coeficiente de mortalidade perinatal (Tabela 1). 
A Figura 2 ilustra a tendência de declínio dos coeficientes de mortalidade perinatal para cada Macrorregional e respectivas Regionais de Saúde constatando-se declínio em todas as RS, destacando-se Foz do Iguaçu em primeiro lugar (Macrorregional Oeste), seguida de Irati e União da Vitória (Macrorregional Leste) e Campo Mourão (Macrorregional Noroeste).

A Figura 3 representa o mapa temático do estado do Paraná por Regionais de Saúde mostrando a distribuição espacial dos CM perinatal, fetal e neonatal precoce, para os triênios extremos. Observa-se que as regiões centro-sul e norte do estado foram as que apresentavam os coeficientes mais elevados no primeiro triênio. No segundo triênio o mapa está mais claro mostrando coeficientes, em geral, mais baixos destacando ainda a $5^{\mathrm{a}} \mathrm{RS}$ de Guarapuava, a $21^{\mathrm{a}}$ de Telêmaco Borba e a $19^{a}$ de Jacarezinho com valores mais elevados, tanto para coeficiente fetal como para o neonatal precoce.
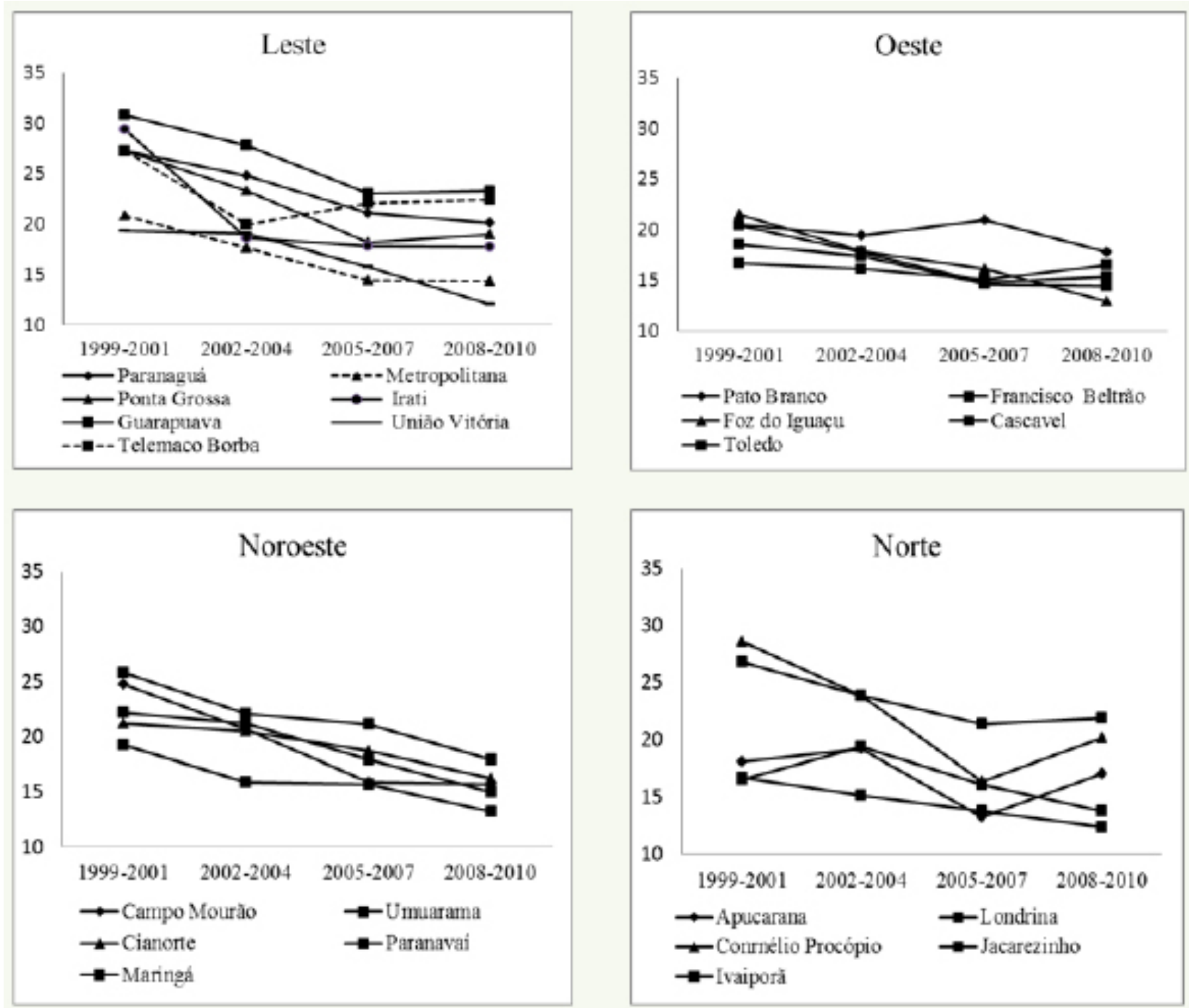

Figura 2 - Coeficiente de mortalidade perinatal (por 1000 NV e NM), segundo Regional e Macrorregional de Saúde, Paraná, Brasil, 1999 a 2010

\section{DISCUSSÃO}

A análise da mortalidade perinatal (fetal e neonatal precoce) no período de 1999 a 2010 evidenciou declínio dos coeficientes no Estado do Paraná de 22,8 óbitos por mil NV e NM em 1999 para 15,3 em 2010, persistindo, entretanto, taxas elevadas para os CM fetal e neonatal precoce, ratificando outros estudos com resultados semelhantes ${ }^{(11-12)}$

Resultados apresentados pelo Ministério da Saúde apontam que, embora o Brasil ainda apresente coeficientes elevados, estes reduziram $16 \%$, de 25,6 em 2000 para 21,5 óbitos por mil NV e NM em 2010 ${ }^{(6)}$. Quando comparados aos resultados de outros países, o Estado do Paraná com 15,3 óbitos por mil NV e NM apresenta 


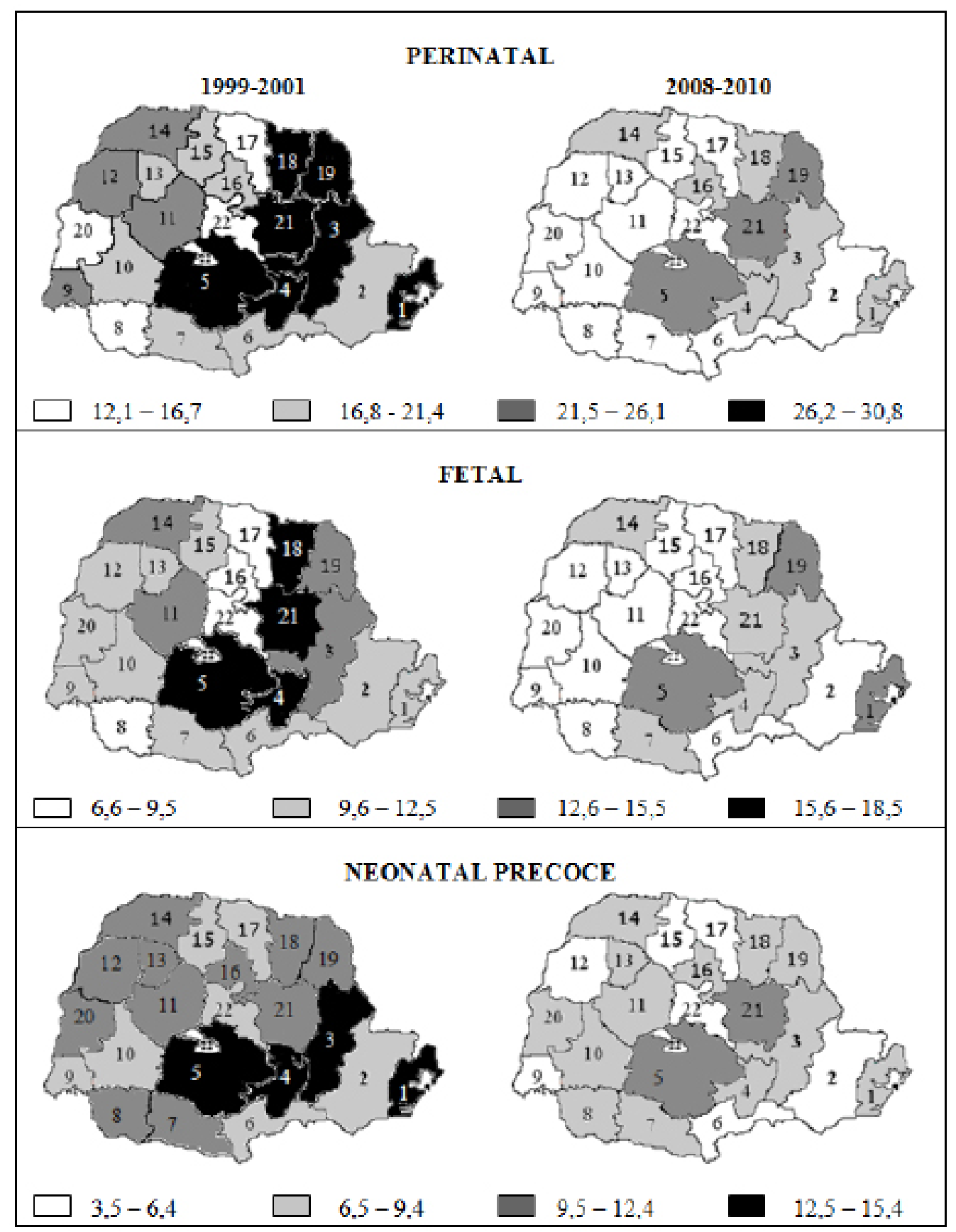

Figura 3 - Coeficiente de mortalidade perinatal segundo componentes e Regionais de Saúde. Paraná, Brasil, 1999-2001 e 2008-2010

melhores resultados para a mortalidade perinatal em relação aos países do continente africano e asiático com coeficientes médios de 60 óbitos por mil NV e NM, mas valores superiores aos países da Europa com média de 8,0 óbitos perinatais por mil NV e NM em 2004(13).

Enquanto o CM neonatal precoce na Região Sul era de 5,9 óbitos por mil NV, o Estado do Paraná apresentou 6,8 óbitos por mil NV em 2010. Resultados mais positivos foram encontrados no Rio Grande do Sul com coeficiente de 5,5 óbitos por mil NV, e valores mais elevados no Estado da Bahia com coeficiente de 12,8, e no Maranhão com 12,7 óbitos por mil $\mathrm{NV}^{(6)}$. O Brasil apresentou redução dos CM neonatal precoce de 13,4 para 8,7 óbitos por mil NV de 2000 a $2010^{(6)}$. Essa redução pode ser atribuída à disponibilidade de tecnologias avançadas para o atendimento aos nascidos vivos graves e prematuros, capazes de promover o restabelecimento da saúde dos neonatos ou prolongar o tempo de vida dos mesmos ${ }^{(11)}$. Já para o componente da mortalidade fetal neste estudo houve tendência decrescente no Estado do Paraná, com redução de 30\% entre 1999 e 2010, redução que pode ser atribuída também à melhoria da qualidade de informação deste indicador, embora algumas variáveis apresentem falhas no registro de dados, interferindo no resultado final. 
Ainda é preocupante o período de 2006 a 2010, no qual os óbitos fetais se mantiveram relativamente estáveis, com coeficientes oscilando de 8,7 a 8,6 óbitos por mil NV e NM.

Apesar do decréscimo que o $\mathrm{CM}$ fetal vem apresentando, é importante destacar que as causas dos óbitos fetais são em sua maioria potencialmente evitáveis ${ }^{(14)}$. No Brasil em 2007, de todos os óbitos fetais registrados, 29,4\% ocorreram em fetos com mais de 2,500g, morte por sífilis congênita ou asfixia intraparto(7). Estimase ainda que ocorram no mundo três milhões de natimortos de terceiro trimestre a cada ano, e destes $98 \%$ ocorrem em países de baixa e média renda, e mais de um milhão ocorre no período intraparto $^{(3)}$.

Os CM fetal apresentaram valores elevados para algumas RS, como a $1^{\mathrm{a}} \mathrm{RS}$ de Paranaguá que registrou aumento de 5,9\% no período, a $8^{\mathrm{a}}$ RS de Francisco Beltrão com aumento de $12,3 \%$ e a $16^{\mathrm{a}}$ RS de Apucarana com aumento de $12,2 \%$. É importante ressaltar que essa variação positiva pode refletir em melhoria de qualidade do registro nos Sistemas de Informações. Mas é necessário aprimorar a qualidade do cuidado à gestante e ao recém-nascido, visto que a morbimortalidade neonatal precoce e a mortalidade fetal compartilham circunstâncias e etiologias semelhantes e são, em sua maioria, consideradas evitáveis, embora muitas vezes sejam vistas como menos importantes.

Observou-se ainda que os CM perinatal, fetal e neonatal precoce mais altos se concentraram em regiões do Estado com indicadores socioeconômicos e grau de desenvolvimento inferiores, como a RS Guarapuava que apresentou os piores valores para a mortalidade perinatal nos dois triênios extremos (coeficientes de 30,8 e 23,3, óbitos perinatais por mil nascimentos), e mais elevados do que os observados em outras RS também no período fetal (18,2 e 12,9 óbitos fetais por mil nascimentos). Em estudo realizado para analisar indicadores da saúde materno infantil no Estado do Paraná, também foram encontrados resultados semelhantes para a RS de Guarapuava, com aglomerados de municípios com mais de $50 \%$ de mães com baixa cobertura de pré-natal, fator determinante para a maior probabilidade de ocorrência de mortalidade perinatal ${ }^{(15)}$.

O óbito perinatal é um importante indicador de saúde que subsidia a avaliação da qualidade da assistência prestada à mulher e à criança, e sofre influência também do grau de desenvolvimento econômico e social da população e do local de estudo. Uma das prováveis explicações para os altos CM perinatal e seus componentes nas mesmas RS do Estado, pode residir nas especificidades das características dessas regiões, onde predominam municípios com baixo e médio grau de desenvolvimento socioeconômico ${ }^{(15)}$. Da mesma forma podem sinalizar, indiretamente, deficiência no acesso aos serviços de saúde e na efetividade das ações ofertadas à gestante durante o pré-natal e parto.

Em relação ao $\mathrm{CM}$ no período neonatal precoce, destacou-se a $21^{\mathrm{a}} \mathrm{RS}$ Telêmaco Borba que apresentou aumento no período de estudo. Assim como para Guarapuava, é necessário maior atenção para essa RS para detectar os fatores que estão influenciando esses indicadores de saúde, considerando que a mortalidade nos primeiros dias de vida expressa complexa conjunção de fatores biológicos, socioeconômicos e assistenciais, sendo esses últimos relacionados à atenção à gestante e ao recém-nascido ${ }^{(16)}$.

Sabe-se que à medida que melhoram as condições sociais, econômicas, o acesso a serviços básicos de saneamento e a serviços de saúde, as mortes infantis no período neonatal tardio e pós-neonatal diminuem e os óbitos na primeira semana, e predominantemente nas primeiras horas de vida, passam a ser os mais freqüentes. Portanto, é necessário aprimorar ainda mais a assistência à saúde da gestante durante o período pré-parto, parto e o atendimento imediato à criança no nascimento e berçário considerando sua estreita relação com a mortalidade perinatal ${ }^{(17)}$.

Uma estratégia para monitorar a mortalidade infantil e fetal foi a implantação, pelo Ministério da Saúde, dos Comitês de Prevenção da Mortalidade Infantil e Fetal. Os comitês têm por objetivo elucidar as circunstâncias da ocorrência dos óbitos infantis e fetais, identificando os fatores de risco, atribuindo a evitabilidade e propondo medidas de melhoria da qualidade da assistência à saúde ${ }^{(1)}$. O Paraná conta com um Comitê Estadual de Prevenção da Mortalidade Infantil e Fetal, 22 Comitês Regionais e municipais, e ainda conta com Comitês hospitalares. O trabalho dos Comitês constitui instrumento de avaliação da assistência de saúde, contribui para o melhor conhecimento sobre os óbitos infantis e fetais subsidiando as políticas públicas e ações de intervenção ${ }^{(18)}$.

Mesmo com o declínio do CM perinatal e de seus componentes, e não obstante os esforços do governo Estadual e da Secretaria de Estado da Saúde, este estudo mostrou que ainda 
persistem óbitos que poderiam ser evitados, em especial em algumas regiões do Estado do Paraná. A implantação de clínicas de Saúde da Mulher e da Criança, o Programa Nascer no Paraná e, recentemente, a implantação da Rede Mãe Paranaense, em 2012 $2^{(18)}$, são algumas das estratégias para qualificar a assistência à gestante, estabelecer redes de atenção, com objetivo de diminuir a morbimortalidade materna e infantil.

\section{CONSIDERAÇÕES FINAIS}

Este estudo analisou a mortalidade perinatal e seus componentes segundo Macrorregionais e RS do Estado do Paraná no período de 1999 a 2010. Redução mais importante foi encontrada para o componente neonatal precoce em relação ao componente fetal e observou-se que, a partir de 2007, os coeficientes permaneceram relativamente estáveis. Entre os componentes da mortalidade perinatal, houve aumento do CM fetal nas RS de Paranaguá, Francisco Beltrão e Apucarana; a RS de Toledo apresentou aumento do CM neonatal precoce e a RS de Guarapuava apresentou os piores valores, para o óbito perinatal e fetal, nos dois triênios extremos.

Os resultados evidenciaram variação dos $\mathrm{CM}$ entre as Regionais e Macrorregionais de Saúde, sinalizando a necessidade de análises mais acuradas direcionadas para cada Regional de Saúde isoladamente, buscando associação da mortalidade perinatal a fatores socioeconômicos, demográficos, de atenção à saúde e de organização de serviços de saúde. Como existe heterogeneidade no perfil da população entre as regiões, estudos futu ros devem ser realizados para compreender como esses fatores de risco estão distribuídos nas diferentes regiões do estado do Paraná, e se as variações nos coeficientes de mortalidade perinatal observadas neste estudo podem ser atribuídas a essas características locais e quais são mais relevantes. Portanto, é importante que as equipes de trabalhadores das Regionais de Saúde do Paraná incentivem e contribuam para a discussão nos municípios sobre os coeficientes e metas a serem alcançados e ainda sobre a necessidade de melhorar o acesso e a qualidade da atenção ao pré-natal e ao recémnascido de risco.

Vale destacar que existem algumas limitações que devem ser consideradas para a interpretação dos resultados deste estudo. Existe a possibilidade de que os valores dos coeficientes de mortalidade perinatal sejam influenciados pelo diferencial de qualidade dos registros dos dados de nascimento e de óbitos nos hospitais e outros serviços de saúde e também nos setores de epidemiologia dos municípios de residência da mãe onde esses dados são digitados. Esses fatores determinam em diferentes gradientes a qualidade do SIM e do Sinasc para cada localidade.

Neste contexto, o estudo da mortalidade perinatal de forma localizada pelos serviços de saúde é de extrema importância já que as equipes multiprofissionais são responsáveis pelo desenvolvimento de ações de promoção da saúde da mulher e da criança, tanto no cuidado à população como na organização dos serviços e estabelecimento de redes de atenção à gestante e recém-nascido. Essas ações incluem acompanhamento no pré-natal, parto e puerpério, ações de educação para a saúde, bem como ações específicas de prevenção da mortalidade perinatal como a captação precoce de mulheres gestantes e recém-nascidos de risco, em áreas de abrangência da Estratégia Saúde da Família.

São necessários ainda novos estudos direcionados ao conhecimento do perfil da morbimortalidade perinatal, principalmente análises dos diferenciais desses riscos à saúde da criança entre as Regionais de Saúde. É necessário ainda ter ciência dos motivos da estabilização dos valores dos coeficientes de mortalidade perinatal a partir de 2007, e determinar as ações que podem ser aplicadas visando à diminuição desses coeficientes.

\section{REFERÊNCIAS}

1. Ministério da Saúde (BR). Secretaria de Atenção à Saúde. Secretaria de Vigilância em Saúde. Manual de vigilância do óbito infantil e fetal e do comitê de prevenção do óbito infantil e fetal. Brasília, DF; 2009.

2. Organização Mundial da Saúde (OMS). Classificação estatística internacional de doenças e problemas relacionados à saúde. 1. ed. rev. São Paulo: Centro Colaborador da OMS para classificação de Doenças em Português; 2007.

3. Goldenberg RL. Stillbirth: the vision for 2020. Lancet. 2011; 337(9779):1798-805.

4. Cousens S, Blencowe $\mathrm{H}$, Stanton C, Chou D, Ahmed S, Steinhardt L, et al. National, regional, and worldwide estimates of stillbirth rates in 2009 with trends since 1995: a systematic analysis. Lancet. 2011; 377(9779):1319-30.

5. Drumond EF, Machado CJ, França E. Óbitos neonatais precoces: análise de causas múltiplas de morte pelo método Grade of Member ship. Cad. Saúde Pública. 
2007; 23(1):157-66.

6. Ministério da Saúde (BR). Secretaria de Vigilância em Saúde. Mortalidade Infantil no Brasil: Tendência, componentes e causa de morte no período de 2000 a 2010. Saúde Brasil 2011: uma análise da situação de saúde e a vigilância da saúde da mulher. Brasília; 2012.

7. Paris GF, Mathias TAF. Fatores associados à ausência de dados no sistema de informações sobre nascidos vivos em condições desfavoráveis no nascimento. Cien. Cuid. Saúde. 2012; 11(4):673-80.

8. Victora CG, Aquino EML, Leal MC, Monteiro CA, Barros FC, Szwarcwaldet CL. Saúde de mães e crianças no Brasil: progressos e desafios. Lancet. 2011; 377(1):3246.

9. Secretaria de Estado da Saúde do Paraná (PR). Rede mãe paranaense. [Internet]. 2012 [acesso em 12 out 2013]. 29 p. Disponível em: http://www.saude.pr.gov.br/ arquivos/File/APSUS_-_Ed._Permanente/Oficina_02/ Rede_Mae_Paranaense.pdf

10. Rede Interagencial de Informações para Saúde. Indicadores básicos para saúde no Brasil: conceitos e aplicações. Brasília: Organização Pan-Americana da Saúde (OPAS); 2008.

11. Martins EF, Lana FC, Maria E. Tendência da mortalidade perinatal em Belo Horizonte, 1984 a 2005. Rev. Bras. Enferm. 2010; 63(3):446-51.

12. Barreto JOM, Nery IS, Mendes YMMB. Mortalidade perinatal: uma análise com enfoque na evitabilidade. Cogitare enferm. 2011; 16(1):88-95.

13. Ahman E, Zupan J. Neonatal and perinatal mortality: country, regional and global estimates 2004. Geneva: World Health Organization; 2007.

14. Martins EF, Rezende EM, Lana FCF, Souza KV. Óbitos perinatais investigados e falhas na assistência Hospitalar ao parto. Esc Anna Nery. 2013; 17(1):38-45.

15. Melo EC, Mathias TAF. Distribuição e autocorrelação espacial de indicadores da saúde da mulher e da criança, no Estado do Paraná, Brasil. Rev. Latino-am. Enfermagem. 2010; 18(6):1177-86.

16. Soares ES, Menezes GMS. Fatores associados à mortalidade neonatal precoce: análise de situação no nível local. Epidemiol. Serv. Saúde, 2010; 19(1):51-60.

17. Silva ZP, Almeida MF, Ortiz LP, Alencar GP, Alencar AP, Schoeps D, et al. Morte neonatal precoce segundo complexidade hospitalar e rede SUS e não SUS na Região Metropolitana de São Paulo, Brasil. Cad. Saúde Pública, 2010; 26(1):123-4.

18. Secretaria de Estado da Saúde do Paraná (PR). Seminário Integrado do Paraná: 20 anos do Comitê de Prevenção de Mortalidade Materna - 10 anos de Mortalidade Infantil. Curitiba, 2009. 